\title{
Cross-Layer Analysis of Multi-Static RFID Systems Exploiting Capture Diversity
}

\author{
Roberto Valentini, Member, IEEE, Piergiuseppe Di Marco, Member, IEEE, Roberto Alesii, \\ and Fortunato Santucci, Senior Member, IEEE,
}

\begin{abstract}
In this paper, we propose a framework for crosslayer analysis of multi-static passive RFID systems. The model takes into account details of the shared wireless channel, including fading and capture effect, whereas, at the medium access control (MAC) layer, the anti-collision mechanism proposed in the EPC Generation 2 standard is taken as a reference. To address the complexity of the system model, we rely on a semi-analytical approach, that combines a moment matching approximation method to abstract the physical layer and MonteCarlo simulations to describe the MAC dynamics. Furthermore, based on the space diversity feature offered by the multi-static settings, we introduce the concept of capture diversity and propose a modification to the standard to fully support this form of diversity. Numerical results show the impact of deployment conditions and the relative positions of interrogator, tags, and detection points on the performance of tags' identification. We show how the number of detection points impacts the system performance under various channel conditions and MAC parameters' settings. Finally, we validate the proposed update of the MAC protocol, showing substantial performance improvement with respect to the standard collision resolution policy.
\end{abstract}

\section{INTRODUCTION}

Backscattering communication has been recently attracting a renewed interest in the context of pervasive wireless connections, and it is seen as a fundamental technology to enable Internet of Things (IoT) applications [1]. Although backscatter radios are currently exploited for passive sensing, asset tracking, and tag identification, employing Ultra High Frequency (UHF) Radio Frequency Identification (RFID) techniques, recent research trends envisage backscattering technology as a relevant component of future generation cellular systems (beyond 5G), in the context of Ultra-Low-Power Machine Type Communications, with applications in logistics, intelligent transportation systems, and health-care sector [2]. Moreover, the ability to identify and locate objects by the use of passive backscattering devices has a great potential to support asset tracking and contact tracing scenarios in medical emergencies to control epidemic outbreaks.

The simplest RFID scenario is a centralized (i.e., monostatic) architecture consisting of an interrogator (i.e., the reader), with physically co-located transmitting and receiving antenna, and a population of passive transponders (i.e., the

R. Valentini, P. Di Marco and F. Santucci are with the Department of Information Engineering, Computer Science and Mathematics and Center of Excellence EX-EMERGE, University of L'Aquila, L'Aquila, 67100 Italy, E-mail: \{roberto.valentini - piergiuseppe.dimarco — fortunato.santucci\} @ univaq.it.

R. Alesii is with the Department of Information Engineering, Computer Science and Mathematics and Center of Excellence DEWS, University of L'Aquila, L'Aquila, 67100 Italy, E-mail: roberto.alesii@univaq.it tags) that has to be identified by the interrogator. The passive nature of the tags (i.e., the absence of a dedicated power source) inherently imposes a very simple design that is based on RF energy scavenging and backscattering communication. The interrogator transmits a continuous wave signal to the tags that in turn scavenges a portion of the incident power to operate an antenna's load impedance switching modulator [3].

It turns out that RFID systems resort on a mono-static radar-like communication over a very asymmetrical link. The asymmetry comes from the fact that the RF power required at a tag to enable backscattering (i.e., the harvesting circuit sensitivity threshold) is order of magnitudes higher than the minimum backscattered power that needs to be received by the reader to process information (i.e., the receiver sensitivity of the reader). Therefore, the region of active tags participating in the identification process is confined in a limited area (typically a few meters) around the interrogator [4], but the backscattered signal can be detected in a larger region.

Moreover, the inherent simplicity of passive tags' architecture poses substantial limitations to the design of the system protocol stack. For instance, the tags' inability to perform channel sensing restricts the applicability of complex collision resolution mechanisms. In general, channel access contention is driven by the interrogator and is based on simple ALOHA protocols, such as the anti-collision scheme defined by the EPC Generation 2 (Gen 2) standard [5], which is characterized by very low overhead and high energy efficiency.

Despite the inherent low cost and low complexity constraints, that make RFID systems appealing for a variety of IoT applications, traditional centralized RFID architectures often fail to cope with the target application requirements. To overcome the limitations of centralized systems, distributed (i.e., multi-static) architectures have been considered as a viable solution to extend the capabilities of passive identification [6]-[9]. Albeit the system complexity increases, distributed systems provide extended coverage and may substantially improve the identification performance if compared to their centralized counterpart.

The last concern is even more evident when the underlying propagation channel characteristics are taken into account in the analysis and design of RFID communication protocols. Indeed, on the one hand, the fading affecting the interrogation signals has a negative effect on the forward link, causing tags powering failures. On the other hand, powered tags may benefit of channel fading in the backscattering links by exploiting the capture effect, where interfering tags' replies, which generally result in a collision, can be resolved as a 
successful identification despite the interference. In distributed systems, the achieved performance improvement may be even more relevant since the capture probability can vary at different detection locations. Consequently, multiple tags may be identified simultaneously during collision events.

\section{A. Related Work}

Motivated by the renewed visibility of backscattering communications in the context of beyond $5 \mathrm{G}$ and massive IoT arenas, many recent studies explore physical layer solutions to improve communication performance and extend the applicability of traditional RFID paradigms. Great attention is deserved to emerging ambient backscattering approaches, where backscattering modulation relies on existing RF signals without the need of dedicated transmitters [10], [11]. Simultaneously, multi-antenna solutions, that are widely used in traditional one-way wireless channels, have been considered to improve performance and increase capacity of backscattering communication systems. Recent advancements in this direction propose Multiple Input Multiple Output (MIMO) architectures considering multiple antennas at either tag side or reader side. Specifically, traditional space-time coding (STC), beamforming-like techniques and transmit diversity for the forward link, have been adapted for backscatter MIMO systems [12]-[17].

Regarding cross-layer analysis of mono-static RFID systems, several modeling and optimization approaches that jointly consider channel statistics and Medium Access Control (MAC) dynamics, have been proposed. In [18], a characterization of the channel losses in RFID systems is provided, but only on single transmissions. [19]-[22] propose the analysis and optimization of slotted ALOHA channel access schemes in the presence of the capture effect [23]. However, in most prior studies the capture probability is often assigned as a model parameter, thus resulting in a significant simplification of the underlying channel. The capture effect and the effect of spatial correlation on Gen 2 based mono-static RFID systems has been studied in our prior work [24], while in [25] the first and second order statistics of the backscattering links are derived for both time-invariant and time-variant channel statistics and used to build a Markov chain model of an ALOHA based collision resolution scheme. A theoretical analysis of throughput and bit error rate for multi-tag systems, including propagation channel statistics, has been performed in [26].

Prior work on multi-static RFID systems mainly focuses on the derivation of proper readers' activation policies aimed at reducing interference among readers, thus avoiding colliding interrogations. Indeed, in the presence of multiple readers that concurrently participate in the tag identification problem, collisions in the interrogation phase may substantially increase the identification time. The seminal work in [27] exploits graph coloring theory to derive a distributed scheduling algorithm that allows to mitigate interference among readers. In [28], [29] the authors deal with the derivation of a stable readers scheduling algorithm designed for dynamic environments, where the number of arriving tags in the interrogation area changes over time. In [30] a heuristic multi-channel scheduling algorithm for multiple readers coordination is derived with the advantage of being adaptable to existing multiple access schemes for single reader scenarios. In [31], an optimizationbased distributed interrogation scheme is proposed in order to avoid unpractical centralized coordination mechanisms among readers. In [32], an approach based on readers cooperation is proposed to handle collisions. The study in [33] proposes an approach based on Q-learning, which attempts to minimize interference by learning the collision patterns of the readers and by properly allocating frequency resources to ensure neighboring readers do not experience interference. Finally, the recent work in [34] proposes a novel reader scheduling policy that substantially outperforms state of the art solutions. Nevertheless, all the aforementioned studies neither consider the physical layer nor address the underlying propagation channel. More importantly, the mentioned literature does not deal with the receiving redundancy offered by the multi-reader architecture. Furthermore, the passive nature of RFID tags, which may induce energy outage, is not explicitly modeled.

The recent study in [35] considers a multi-static system consisting of a set of interrogators and a single detection point. The proposed framework includes accurate models for the physical layer and the propagation channel. A comprehensive analysis is conducted, which includes the derivation of Bit Error Rate (BER) performance bounds, information and energy outage probabilities. Finally, in [36] a multi-static architecture is proposed, where MAC layer operations are distributed over a set of networked Software Defined Radio (SDR) transmitters. The proposed solution envisages the convergence of Gen 2 RFID with Ethernet networks or current and future cellular systems. However, the studies mentioned above do explicitly include neither receiver diversity nor cross-layer performance evaluation of the considered RFID architectures.

\section{B. Contributions and Paper Organization}

In this paper, we propose a general cross-layer framework that considers simultaneously anti-collision mechanism and propagation characteristics in a multi-static Gen 2 RFID system with a single interrogator and multiple networked receivers. Specifically, we focus on the combination of receiving diversity and channel contention mechanism that give rise to the so-called capture diversity, first introduced in our prior work [37]. In particular, we show how different samples of fading and interference at different receivers can be exploited in multi-static systems to: $i$ ) resolve simultaneous tags' responses during collision events; $i$ ) reduce the overall collision rate and improve the identification performance.

The contributions of this work are summarized as follows.

- We present a framework for the characterization of multiple access interference and capture effect to study the performance of passive tags' identification. The model includes channel and propagation characteristics and their impact on tags' powering under the assumption of lognormal fading statistics. To this aim we adopt a generalized Moment Matching Approximation (MMA) approach that was first proposed in [38] and here extended to the multivariate analysis. 
- We leverage on the analysis in our prior study in [39] to detail the peculiar features of the capture probability in multi-static RFID systems and the relative impact on MAC layer performance, considering the Gen 2 collision resolution protocol. To reduce the complexity of a pure analytic framework, we employ a semi-analytic approach, where the analytical derivation of the equivalent channel model is coupled with MAC layer Monte-Carlo simulations. This allow to simplify the analysis of interactions between the propagation channel impairments and the dynamics of the channel access scheme.

- We propose and validate modifications to the Gen 2 channel access arbitration to support and exploit the presence of multiple detection points. A detailed simulation study is provided to quantify the substantial identification performance improvement induced by capture diversity in the considered multi-static settings when compared to the centralized architecture. Moreover, the effect of channel severity, MAC parameters and number of deployed detection points in the system is analyzed.

We remark that we do not consider a multi-reader scenario consisting of independent interrogators as in [27], [35], [36]. Instead, we focus on the inherent properties offered by the presence of different detection points and the randomness in the channel contention scheme. This scenario could be interpreted as a snapshot of a multi-reader with scheduled interrogations, where in a given phase a reader interrogates and the other readers act as detection points.

Furthermore, the proposed standard modification does not affect the system complexity and does not require communication among detection points. Indeed, in the considered settings, the receivers independently perform tags' identification and only need to communicate the result of the identification process to the interrogator.

The rest of this paper is organized as follows. In Section II we describe the considered RFID multi-static architecture and the Gen 2 anti-collision scheme. In Section III we introduce the system model focusing on the propagation scenario and its impact on the MAC layer dynamics. Furthermore, we characterize multiple-access interference via MMA. In Section IV we introduce capture diversity and discuss how it can be supported by the Gen 2 standard. In Section V numerical results are provided. Finally, Section VI concludes the paper.

Notation: random variables are denoted by sans-serifstyle upper-case letters (e.g., X). Sets are denoted by calligraphic upper-case letters $($ e.g., $\mathcal{X})$ unless otherwise stated. The operators $\operatorname{Pr}(\cdot), E[\cdot]$ and $\mathbb{1}_{(\cdot)}$ represent probability measure, expectation and indicator function of an the event, respectively.

\section{SYSTEM SCENARIO}

\section{A. Multi-static Architecture}

We consider the RFID system depicted in Fig. 1, consisting of a set of passive tags $\mathcal{T}$, with $|\mathcal{T}|=N$, an interrogator and a set of networked backscattering receivers (i.e., detection points) $\mathcal{R}$, with $|\mathcal{R}|=R$. We assume that the interrogator transmit power, $P_{\mathrm{tx}}$ is fixed and we denote with $\mathrm{P}_{f, k}$ the

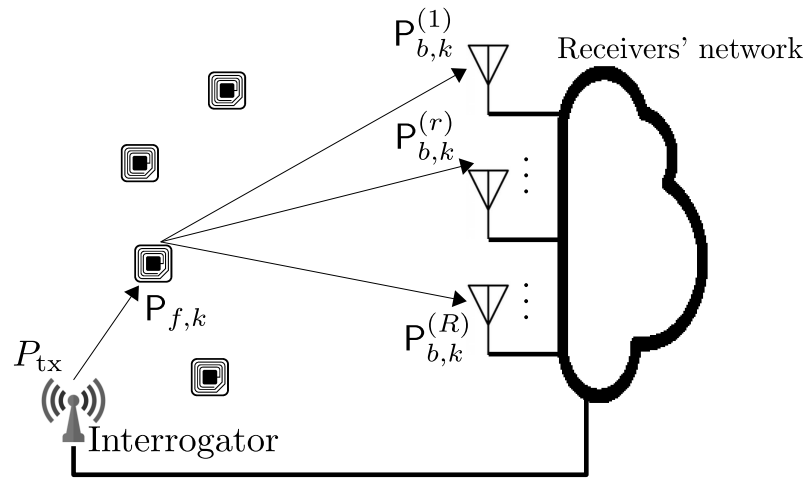

Fig. 1. Multi-static architecture.

power received at the $k$-th tag. The interrogator drives the identification process by providing command signals that are also used by the tags to extract the energy required to power-up the backscattering modulator. The signals backscattered from the tags are detected by the readers' network at different spatial locations. The power scattered by the $k$-th tag and received at the $r$-th detection point is denoted by $\mathrm{P}_{b, k}^{(r)}$. Furthermore, we assume that information about the identification of a tag is available immediately at the interrogator. This can be achieved by a high speed link between the detection point and the interrogator, where the latency is negligible compared to the tags' identification time. The characterization of this high speed network is however beyond the scope of this paper and is not explicitly included here.

\section{B. EPC Global Gen 2 Protocol}

The identification process in most commercial RFID systems is implemented at MAC layer, where a suitable collision resolution protocol is needed to regulate the channel contention among tags. The reference standard for single-reader RFID systems operating in the RF frequency range of $860-$ $960 \mathrm{MHz}$ is the Gen 2 [5], as part of the ISO/IEC 18000-6 standard.

According to the MAC specifications, an interrogation round is defined as a sequence of consecutive commands and responses that are issued by the interrogator and replied back by the tag in order to transmit back the identifier of the tag. A sequence of consecutive interrogation rounds forms an interrogation session. At the beginning of the session, the reader transmits a Query command with an integer parameter $Q$, asking every tag to generate a 16-bits random number (RN16) according to a uniform distribution and to mask $Q$ bits of the generated RN16 to form their slot counter. At each slot, the reader sends QueryRep commands which forces the tags decrease their slot counter.Tags respond with their RN16 when their counter counts down to 0 , forming a framed slotted ALOHA multi-access system over a duration of $2^{Q}$ slots.

Assuming that a single tag is identified, the reader responds by sending an acknowledgement (ACK) including the received RN16. The tag can then transmit its packet. If the packet is not detected, the reader issues a NACK to notify the tag that 
remains active in the arbitration. If acknowledged, the tag will not respond to other Query/QueryRep/QueryAdj commands in the same session.

During the evolution of the identification procedure, the frame length is adapted "greedly" in response of idle, collided or successful identification according to an adaptive procedure known as $Q$-Algorithm [5]. Specifically, the interrogator tracks a floating point variable, $q_{F P}(t)$, that is updated on a slot basis as $q_{F P}(t+1)=q_{F P}(t)-c$ if the slot is idle, $q_{F P}(t+$ $1)=q_{F P}(t)+c$ if a collision is detected, or left unchanged if a tag is correctly identified.

Then, the new value of $Q$ for slot $t+1$ is obtained as $Q(t+1)=\left\lfloor q_{F P}(t+1)\right\rceil$, where $\lfloor\cdot\rceil$ is the nearest integer function. As the $Q$ value changes, the interrogator broadcasts a QueryAdj command instructing nodes to reset the backoff counter consistently with the updated value of the frame length $2^{Q}$. The granularity parameter $c$, with $0.1 \leq c \leq 0.5$, can be used to tune the reactivity of the algorithm in response to collisions and idle slots. Specifically, by increasing the value of $c$, the algorithm reacts more aggressively to subsequent colliding and idle slots, with more frequent adaptations of the $Q$ parameter. The identification session ends when all the tags in the population are identified.

\section{Multi-Static RFID System Model}

\section{A. Channel Model}

Different from traditional one-way channels, backscattering channels exhibit composite fading effects, given that the received signal at a generic detection point is affected by the product of two cascade fading components. Motivated by static or slowly moving identification scenarios, we assume that the communication occurs over a slow varying channel, where the fading remains constant within a time interval $\Delta T$ and we assume that the fading experimented at subsequent intervals is independent from each other.

Under this settings, the power received by the $k$-th tag through the forward link (i.e., from the interrogator) within a coherence interval $\Delta T$ can be expressed as

$$
\mathrm{P}_{f, k}=P_{\mathrm{tx}} \alpha_{k} e^{\mathrm{S}_{f, k}}
$$

with $k \in \mathcal{T}$, where $\alpha_{k}=c_{0} / d_{f, k}^{k_{0}}, d_{f, k}$ is the distance between the interrogator and the $k$-th tag, $c_{0}$ is the frequency-dependent path-loss at reference distance of 1 meter and $k_{0}$ is the pathloss exponent. The fading is described by the the zero-mean Gaussian random variable $\mathrm{S}_{f, k} \sim \mathcal{N}\left(0, \sigma^{2}\right)$, where $\sigma$ is an indicator of channel asperity.

In order for a tag to be able to activate the backscattering modulator, the received power $\mathrm{P}_{f, k}$ has to be larger than the harvesting circuit sensitivity, which we denote as $\gamma_{h}$. Then, the received power at the $r$-th receiver can be expressed as

$$
\mathrm{P}_{b, k}^{(r)}=\beta_{r, k} \mathrm{P}_{f, k} e^{\mathrm{S}_{b, k}^{(r)}} \mathbb{1}_{\left(\mathrm{P}_{f, k}>\gamma_{h}\right)},
$$

with $r \in \mathcal{R}$. Here, $\beta_{r, k}=c_{0} / d_{b, k}^{k_{0}}$ where $d_{b, k}$ is the distance between the $k$-th tag and the $r$-th detection point and $\mathrm{S}_{b, k}^{(r)} \sim \mathcal{N}\left(0, \sigma^{2}\right)$ describes the fading for the backward link between the $k$-th tag and the $r$-th receiver. Given the physical separation between the transmit and receiving antennas, the fading components on the forward and backward links are here assumed to be independent. Substituting Eq. (1) in Eq. (2), the latter one can be rewritten as

$$
\mathrm{P}_{b, k}^{(r)}=P_{\mathrm{tx}} D_{r, k} e^{\mathrm{Y}_{k}^{(r)}} \mathbb{1}_{\left(\mathrm{P}_{f, k}>\gamma_{h}\right)},
$$

where $D_{r, k}=\alpha_{k} \beta_{r, k}$ and $\mathrm{Y}_{k}^{(r)}=\mathrm{S}_{f, k}+\mathrm{S}_{b, k}^{(r)}$.

Instead, when referring to a mono-static system in which the transmitting and the receiving antenna are physically colocated, the forward and backward channels can be safely assumed to be fully correlated [40] and the received backscattered power at the reader can be expressed as

$$
\mathrm{P}_{b, k}^{(0)}=P_{\mathrm{tx}} D_{0, k} e^{\mathrm{Y}_{k}^{(0)}} \mathbb{1}_{\left(\mathrm{P}_{f, k}>\gamma_{h}\right)},
$$

where, $D_{0, k}=\alpha_{k}^{2}$ and $Y_{k}^{(0)}=2 S_{f, k}$.

In our multi-static scenario we assume that fading components of different links are independent and identically distributed (i.i.d.), that is the random variables $\mathrm{S}_{f, k}$ and $\mathrm{S}_{b, k}^{(r)}$, for $k=1, \ldots, N$ and $r=1, \ldots, M$, are independent and exhibit the same variance. It has to be noted that the receiving antennas separation is much higher than the operative wavelength and the links correlation is expected to be reasonably low. Consequently, the assumption of i.i.d. fading components represents well the case study. Moreover, the choice of considering log-normal channel statistics is motivated by the fact that in many indoor scenarios, log-normal distribution is well suited to describe both large scale and small scale fading phenomena [41]. The inclusion of log-normally distributed multipath fading would not invalidate the proposed channel abstraction. Indeed, and additional log-normal factor in both Eq. (1) and Eq. (2) would result in an overall log-normally distributed fading component with higher variance. Therefore, the effect of small scale fading can be easily described by considering a higher $\sigma$.

\section{B. Medium Access Control Model}

It is worth noting that, due to the fading affecting the forward links, only a subset $\mathcal{A}$ of tags in the population effectively participate to the identification process. Then, with the objective of applying our model to MAC layer performance analysis, a first step is to determine the probability that the set of tags $\mathcal{A} \subset \mathcal{T}$ correctly receives the Query and is able to modulate the backscattered signal. We define the powering event for the tags in $\mathcal{A}$ as $\mathcal{P}_{\mathcal{A}}$ and the powering probability is obtained as

$$
\begin{aligned}
\operatorname{Pr}\left(\mathcal{P}_{\mathcal{A}}\right)= & \prod_{i \in \mathcal{A}} \operatorname{Pr}\left(\mathrm{P}_{f, i}>\gamma_{h}\right) \prod_{j \in \mathcal{T} \backslash \mathcal{A}}\left[1-\operatorname{Pr}\left(\mathrm{P}_{f, j}>\gamma_{h}\right)\right] \\
= & \prod_{i \in \mathcal{A}} \operatorname{Pr}\left(\mathrm{S}_{f, i}>\ln \frac{\gamma_{h}}{\alpha_{i} P_{\mathrm{tx}}}\right) \\
& \times \prod_{j \in \mathcal{T} \backslash \mathcal{A}}\left[1-\operatorname{Pr}\left(\mathrm{S}_{f, j}>\ln \frac{\gamma_{h}}{\alpha_{j} P_{\mathrm{tx}}}\right)\right] \\
= & \prod_{i \in \mathcal{A}}\left[1-F_{\mathrm{S}}\left(\ln \frac{\gamma_{h}}{\alpha_{i} P_{\mathrm{tx}}}\right)\right] \prod_{j \in \mathcal{T} \backslash \mathcal{A}} F_{\mathrm{S}}\left(\ln \frac{\gamma_{h}}{\alpha_{j} P_{\mathrm{tx}}}\right) .
\end{aligned}
$$


where $F_{\mathrm{S}}(x)$ is the Cumulative Distribution Function (CDF) of a zero-mean Gaussian random variable with variance $\sigma^{2}$. The tags in $\mathcal{T} \backslash \mathcal{A}$ are not able to communicate until a new Query command is issued by the interrogator. Clearly, due to tags powering failure, multiple Query commands may be necessary to complete the identification process. Without loss of generality, we refer our analysis to a single identification session.

Upon receiving the Query, each tag independently initializes a slot counter $S N_{k}(0)$ with $k=1, \ldots, N$, which is sampled at random from the interval $\left[0,2^{Q}-1\right]$ (i.e., $S N_{k}(0) \sim \mathcal{U}\left[0,2^{Q}-\right.$ $1]$ ), with $2^{Q}$ referred to as the frame length. In each slot $t=1,2, \ldots$, the interrogator broadcasts a command (i.e., QueryRep) instructing the tags to decrease their slot counters. The tags whose counter $S N_{k}(t)=0$ gain access to the channel and start transmitting.

Denoting by $\mathcal{I}(t) \subset \mathcal{A}$ the set of replying nodes in each slot $t$, that is $\mathcal{I}(t)=\left\{k: S N_{k}(t)=0\right\}$, with cardinality $n_{\mathcal{I}}=|\mathcal{I}(t)|$, the following mutually exclusive outcomes can be experimented on the detectors' side:

a) Idle Slot: $n_{\mathcal{I}}=0$, that corresponds to the case where no tag has a null slot counter, and thus no transmission occurs in the slot;

b) Single Reply: $n_{\mathcal{I}}=1$, that corresponds to the case where only one tag has a slot counter set to zero. The received signal is correctly detected by the $r$-th receiver if the power $\mathrm{P}_{b, k}^{(r)}$ is larger than the receiver sensitivity threshold, which we denote as $\gamma_{d}$. Then, conditioned to $\mathcal{P}_{\mathcal{A}}$, the probability for a single replying tag to be successfully identified is $\operatorname{Pr}\left(\mathrm{P}_{b, k}^{(r)}>\gamma_{d} \mid \mathcal{P}_{\mathcal{A}}\right)$ and, due to the independence between fading components, we obtain

$$
\begin{aligned}
\operatorname{Pr}\left(\mathrm{P}_{b, k}^{(r)}>\gamma_{d} \mid \mathcal{P}_{\mathcal{A}}\right) & =\operatorname{Pr}\left(\mathrm{P}_{b, k}^{(r)}>\gamma_{d} \mid \mathrm{P}_{f, k}>\gamma_{h}\right) \\
& =\frac{\operatorname{Pr}\left(\mathrm{P}_{b, k}^{(r)}>\gamma_{d}, \mathrm{P}_{f, k}>\gamma_{h}\right)}{\operatorname{Pr}\left(\mathrm{P}_{f, k}>\gamma_{h}\right)} .
\end{aligned}
$$

Then, it may happen that the power at the $r$-th receiver input is below the receiver sensitivity threshold, thus yielding a single reply slot to be interpreted as an idle slot. On the contrary, when referring to a mono-static system with fully correlated fading components, we have $\operatorname{Pr}\left(\mathrm{P}_{b, k}^{(0)}>\gamma_{d} \mid \mathrm{P}_{f, k}>\gamma_{h}\right)=1$ whenever $\gamma_{d}<\gamma_{h}^{2} / P_{\mathrm{tx}}$ holds true ${ }^{1}$. The last inequality is inherently met for realistic RFID systems, where the receiver sensitivity threshold $\gamma_{d}$ is much lower that the tags harvesting sensitivity $\gamma_{h}$. Therefore, the $k$-th tag is detected provided that it receives enough power to activate the load modulator.

c) Multiple Reply: $n_{\mathcal{I}}>1$, that corresponds to the case where multiple tags are powered and gain access to the channel in the same slot. In this case, the received backscattered signal consists of the superimposition of multiple interfering replies. Conditioned on $\mathcal{P}_{\mathcal{A}}$, a multiple reply may lead to an idle slot

\footnotetext{
${ }^{1}$ The proof of this condition relies on basic probability theory arguments. Specifically, given the full correlation between the forward and backward links, the conditioned probability can be expressed in terms of a single $\log$-normal random variable. Then, it is straightforward to verify that the conditioned detection probability is equal to one if the mentioned inequality holds.
}

event at the $r$-th receiver with probability

$$
p_{\mathrm{i}}^{(r)}=\operatorname{Pr}\left(\sum_{j \in \mathcal{I}} \mathrm{P}_{b, j}^{(r)}<\gamma_{d} \mid \mathcal{P}_{\mathcal{A}}\right),
$$

which corresponds to the probability that the power of the superimposed signals is below the detection threshold. Observe again that in the above mentioned mono-static setting the probability that mixed signal power is below the receiver sensitivity threshold is zero if the condition $\gamma_{d}<\gamma_{h}^{2} / P_{\mathrm{tx}}$ holds true. In this case a multiple tags' reply cannot be interpreted as an idle slot.

Moreover, due to the so-called capture effect, a tag $k \in \mathcal{I}(t)$ can be successfully identified at the $r$-th receiver despite the interference. A successful identification may occur when the backscattered power from a tag is sufficiently large if compared to the power of the interfering signals. Formally, the $k$-th tag is successfully identified at the $r$-th receiver with probability $p_{c, k}^{(r)}=\operatorname{Pr}\left(\operatorname{SIR}_{k}^{(r)}>\gamma \mid \mathcal{P}_{\mathcal{A}}\right)$, where $\operatorname{SIR}_{k}^{(r)}$ is the Signal-to-Interference Ratio (SIR) for the $k$-th tag at the $r$-th detection point and is expressed as

$$
\operatorname{SIR}_{k}^{(r)}=\frac{\mathrm{P}_{b, k}^{(r)}}{\sum_{j \in \mathcal{I}(t)}^{j \neq k} \mathrm{P}_{b, j}^{(r)}},
$$

and $\gamma>1$ is a receiver-specific parameter that quantifies the detector capability of extracting the desired signal despite the interference. We remark that we neglect the effect of additive noise in the derivation of the capture probability. This is justified observing that the considered system is interferencelimited and the noise contribution can be safely ignored in Eq. (8).

The probability of having multiple reply resolved as a successful identification at the $r$-th detection point is $p_{\mathrm{s}}^{(r)}=\sum_{k \in \mathcal{I}(t)} p_{c, k}^{(r)}$, consequently, the probability that a collision occurs at the $r$-th receiver is expressed as

$$
p_{\mathrm{c}}^{(r)}=1-p_{\mathrm{i}}^{(r)}-p_{\mathrm{s}}^{(r)} .
$$

Note that the distribution of the capture probability is timevarying given the randomness introduced by the channel access protocol. It should also be noted that in the assumption of block fading channel, the distribution of the capture probability for a given $\mathcal{I}(t)$ is constant within the fading coherence interval $\Delta T$. The characterization of the multiple access interference is deferred to Section III-C where the idle, capture and collision probabilities in Eq.s (7) and (9) are also derived.

\section{Multiple-Access Interference Characterization}

In order to study the identification performance for the considered multi-static RFID system, a crucial step is the statistical characterization of the interference that allows to determine the evolution of the collision resolution mechanism implemented at the MAC layer. To this aim, we consider a multiple reply with a generic set of responding tags $\mathcal{I}(t)^{2}$, with $n_{\mathcal{I}}>1$. Since the fading is invariant within a coherence interval $\Delta T$ and given the assumption of block fading we assume that

\footnotetext{
${ }^{2}$ From now on, we suppress the time dependence for notation clarity.
} 
the powering event for a tag does not depend on which slot is selected within an interrogation round. Then, conditioned on the event $\mathcal{P}_{\mathcal{A}}$ that a set $\mathcal{A}$ of tags is able to participate to the identification session, the idle probability at the $r$-th detection point is

$$
\begin{aligned}
p_{\mathrm{i}}^{(r)}= & \operatorname{Pr}\left(\sum_{j \in \mathcal{I}} \mathrm{P}_{b, j}^{(r)}<\gamma_{d} \mid \mathcal{P}_{\mathcal{A}}\right) \\
= & \frac{\operatorname{Pr}\left(\sum_{j \in \mathcal{I}} \mathrm{P}_{b, j}^{(r)}<\gamma_{d}, \mathrm{P}_{f, i}>\gamma_{h}, \forall i \in \mathcal{A}\right)}{\operatorname{Pr}\left(\mathrm{P}_{f, i}>\gamma_{h}, \forall i \in \mathcal{A}\right)} \\
= & \frac{\operatorname{Pr}\left(\sum_{j \in \mathcal{I}} \mathrm{P}_{b, j}^{(r)}<\gamma_{d}, \mathrm{P}_{f, i}>\gamma_{h}, \forall i \in \mathcal{I}\right)}{\operatorname{Pr}\left(\mathrm{P}_{f, i}>\gamma_{h}, \forall i \in \mathcal{I}\right)} \\
= & \frac{\operatorname{Pr}\left(\sum_{j \in \mathcal{I}} D_{r, j} e^{\mathrm{Y}_{j}^{(r)}}<\frac{\gamma_{d}}{P_{\mathrm{tx}}}, \mathrm{S}_{f, i}>\ln \frac{\gamma_{h}}{\alpha_{i} P_{\mathrm{tx}}}, \forall i \in \mathcal{I}\right)}{\prod_{i \in \mathcal{I}}\left[1-F_{\mathrm{S}}\left(\ln \frac{\gamma_{h}}{\alpha_{i} P_{\mathrm{tx}}}\right)\right]},
\end{aligned}
$$

where independence between fading components is invoked for the intermediate steps. Similarly, the capture probability at the $r$-th detection point for the $k$-th tag, with $k \in \mathcal{I}$, conditioned on $\mathcal{P}_{\mathcal{A}}$ is

$$
\begin{aligned}
p_{c, k}^{(r)}= & \operatorname{Pr}\left(\frac{\mathrm{P}_{b, k}^{(r)}}{\sum_{j \in \mathcal{I}}^{j \neq k} \mathrm{P}_{b, j}^{(r)}}>\gamma \mid \mathcal{P}_{\mathcal{A}}\right) \\
= & \frac{\operatorname{Pr}\left(\sum_{j \in \mathcal{I}}^{j \neq k} \frac{\mathrm{P}_{b, j}^{(r)}}{\mathrm{P}_{b, k}^{(r)}}<\frac{1}{\gamma}, \mathrm{P}_{f, i}>\gamma_{h}, \forall i \in \mathcal{A}\right)}{\operatorname{Pr}\left(\mathrm{P}_{f, i}>\gamma_{h}, \forall i \in \mathcal{A}\right)} \\
= & \frac{\operatorname{Pr}\left(\sum_{j \in \mathcal{I}}^{j \neq k} \frac{\mathrm{P}_{b, j}^{(r)}}{\mathrm{P}_{b, k}^{(r)}}<\frac{1}{\gamma}, \mathrm{P}_{f, i}>\gamma_{h}, \forall i \in \mathcal{I}\right)}{\operatorname{Pr}\left(\mathrm{P}_{f, i}>\gamma_{h}, \forall i \in \mathcal{I}\right)} \\
= & \frac{\operatorname{Pr}\left(\sum_{j \in \mathcal{I}}^{j \neq k} \frac{D_{r, j}}{D_{r, k}} e_{j}^{(r)}-\mathrm{Y}_{k}^{(r)}<\frac{1}{\gamma}, \mathrm{S}_{f, i}>\ln \frac{\gamma_{h}}{\alpha_{i} P_{\mathrm{tx}}}, \forall i \in \mathcal{I}\right)}{\prod_{i \in \mathcal{I}}\left[1-F_{\mathrm{S}}\left(\ln \frac{\gamma_{h}}{\alpha_{i} P_{\mathrm{tx}}}\right)\right]} .
\end{aligned}
$$

In order to derive the above probabilities, we first need to calculate the statistics of the random variables $\mathrm{Z}^{(r)}=\sum_{j \in \mathcal{I}} D_{r, j} e^{\mathrm{Y}_{j}^{(r)}}$. To overcome the lack of closed-form expression for the p.d.f. of the weighted sum of log-normal variates, we apply the MMA method, which approximates the statistics of linear combination of log-normal components with a log-normal random variable, $\mathrm{Z}^{(r)} \approx e^{\mathrm{W}^{(r)}}$ such that $\mathrm{W}^{(r)} \sim \mathcal{N}\left(\eta_{\mathrm{W}^{(r)}}, \sigma_{\mathrm{W}^{(r)}}^{2}\right)$, where $\eta_{\mathrm{W}^{(r)}}$ and $\sigma_{\mathrm{W}^{(r)}}^{2}$ are unknown parameters. To obtain $\eta_{\mathrm{W}^{(r)}}$ and $\sigma_{\mathrm{W}^{(r)}}^{2}$, we first compute the first and second order moment of $\mathrm{Z}^{(r)}$ :

$$
\begin{aligned}
\eta_{\mathbf{Z}^{(r)}} & \triangleq E\left[\mathbf{Z}^{(r)}\right]=E\left[\sum_{j \in \mathcal{I}} D_{r, j} e^{Y_{j}^{(r)}}\right] \\
& =\sum_{j \in \mathcal{I}} D_{r, j} E\left[e^{Y_{j}^{(r)}}\right]=e^{\sigma^{2}} \sum_{j \in \mathcal{I}} D_{r, j} . \\
\delta_{\mathbf{Z}^{(r)}} & \triangleq E\left[\mathbf{Z}^{(r)^{2}}\right]=E\left[\sum_{j \in \mathcal{I}} D_{r, j} e^{Y_{j}^{(r)}} \sum_{l \in \mathcal{I}} D_{r, l} e^{Y_{l}^{(r)}}\right] \\
& =\sum_{j \in \mathcal{I}} D_{r, j}^{2} E\left[e^{2 Y_{j}^{(r)}}\right] \\
& +\sum_{j \in \mathcal{I}} \sum_{l \neq j} D_{r, j} D_{r, l} E\left[e^{Y_{j}^{(r)}+Y_{l}^{(r)}}\right] \\
& =e^{4 \sigma^{2}} \sum_{j \in \mathcal{I}} D_{r, j}^{2}+e^{2 \sigma^{2}} \sum_{j \in \mathcal{I}} \sum_{l \neq j} D_{r, j} D_{r, l} .
\end{aligned}
$$

We then impose

$$
\left\{\begin{array} { l } 
{ \eta _ { \mathrm { Z } ^ { ( r ) } } = e ^ { \eta _ { \mathrm { W } ^ { ( r ) } } + \frac { 1 } { 2 } \sigma _ { \mathrm { W } ^ { ( r ) } } ^ { 2 } } } \\
{ \delta _ { \mathrm { Z } ^ { ( r ) } } = e ^ { 2 \eta _ { \mathrm { W } ^ { ( r ) } } + 2 \sigma _ { \mathrm { W } ^ { ( r ) } } ^ { 2 } } }
\end{array} \Rightarrow \left\{\begin{array}{l}
\eta_{\mathrm{W}^{(r)}}=\ln \frac{\eta_{\mathrm{Z}^{(r)}}^{2}}{\sqrt{\delta_{\mathrm{Z}^{(r)}}}} \\
\sigma_{\mathrm{W}^{(r)}}^{2}=\ln \frac{\delta_{\mathrm{Z}^{(r)}}}{\eta_{\mathrm{Z}^{(r)}}^{2}}
\end{array}\right.\right.
$$

Once $\eta_{\mathrm{W}^{(r)}}$ and $\sigma_{\mathrm{W}^{(r)}}^{2}$ are obtained, the probability in Eq. (10) can be approximated as

$$
p_{\mathrm{i}}^{(r)} \approx \frac{\operatorname{Pr}\left(\mathrm{W}^{(r)}<\hat{\gamma}_{d}, \mathrm{~S}_{f, i}>\hat{\gamma}_{h, i}, \forall i \in \mathcal{I}\right)}{\prod_{i \in \mathcal{I}}\left[1-F_{\mathrm{S}}\left(\hat{\gamma}_{h, i}\right)\right]},
$$

where we defined $\hat{\gamma}_{d}=\gamma_{d} / P_{\mathrm{tx}}$ and $\hat{\gamma}_{h, i}=\ln \gamma_{h} / \alpha_{i} P_{\mathrm{tx}}$. The computation of the approximated idle probability in Eq. (14) requires the knowledge of the covariance between $\mathrm{W}^{(r)}$ and $\mathrm{S}_{f, i}$, which we define as $c_{\mathrm{W}^{(r)}, i}=\operatorname{Cov}\left(\mathrm{W}^{(r)}, \mathrm{S}_{f, i}\right)$, for all $i \in \mathcal{I}$. To determine $c_{\mathrm{W}^{(r)}, i}$, we first derive the correlations

$$
\begin{aligned}
\delta_{\mathbf{Z}^{(r)}, i} & \triangleq E\left[\mathbf{Z}^{(r)} e^{\mathrm{S}_{f, i}}\right]=E\left[\sum_{j \in \mathcal{I}} D_{r, j} e^{\mathrm{Y}_{j}^{(r)}} e^{\mathrm{S}_{f, i}}\right] \\
& =\sum_{j \in \mathcal{I}} D_{r, j} E\left[e^{\mathrm{Y}_{j}^{(r)}+\mathrm{S}_{f, i}}\right] \\
& =e^{\frac{3}{2} \sigma^{2}} \sum_{j \in \mathcal{I}}^{j \neq i} D_{r, j}+e^{\frac{5}{2} \sigma^{2}} D_{r, i} .
\end{aligned}
$$

Being $E\left[e^{\mathrm{W}^{(r)}} e^{\mathrm{S}_{f, i}}\right]=E\left[e^{\mathrm{W}^{(r)}}\right] E\left[e^{\mathrm{S}_{f, i}}\right] e^{c_{\mathrm{w}}(r), i}$, with $i \in \mathcal{I}$, we get the covariance between the approximating random variable $\mathrm{W}^{(r)}$ and the fading component on the $i$-th forward link as

$$
c_{\mathrm{W}^{(r)}, i}=\ln \frac{\delta_{\mathrm{Z}^{(r)}, i}}{e^{\eta_{\mathrm{W}^{(r)}}+\frac{1}{2}\left(\sigma_{\mathrm{W}^{(r)}}^{2}+\sigma^{2}\right)}} .
$$

As a second stage we address the statistics of the random variables $\mathrm{X}_{k}^{(r)}=\sum_{j \in \mathcal{I}}^{j \neq k} \frac{D_{r, j}}{D_{r, k}} e^{\mathrm{Y}_{j}^{(r)}-\mathrm{Y}_{k}^{(r)}}$, with $k \in \mathcal{I}$, and we apply the MMA method so that $\mathrm{X}_{k}^{(r)} \approx e^{\mathrm{T}_{k}^{(r)}}$ with $\mathrm{T}_{k}^{(r)} \sim \mathcal{N}\left(\eta_{\mathrm{T}_{k}^{(r)}}, \sigma_{\mathrm{T}_{k}^{(r)}}^{2}\right)$. Following the same approach described before, we derive the moments of $\mathrm{S}_{k}^{(r)}$ :

$$
\begin{aligned}
\eta_{\mathbf{X}_{k}^{(r)}} & \triangleq E\left[\mathbf{X}_{k}^{(r)}\right]=E\left[\sum_{j \in \mathcal{I}}^{j \neq k} \frac{D_{r, j}}{D_{r, k}} e^{Y_{j}^{(r)}-\mathrm{Y}_{k}^{(r)}}\right] \\
& =\sum_{j \in \mathcal{I}}^{j \neq k} \frac{D_{r, j}}{D_{r, k}} E\left[e^{\mathrm{Y}_{j}^{(r)}-\mathrm{Y}_{k}^{(r)}}\right]=\frac{e^{\psi}}{D_{r, k}} \sum_{j \in \mathcal{I}} D_{r, j} . \\
\delta_{\mathbf{X}_{k}^{(r)}} & \triangleq E\left[\mathbf{X}_{k}^{(r)^{2}}\right] \\
& =E\left[\sum_{j \in \mathcal{I}}^{j \neq k} \frac{D_{r, j}}{D_{r, k}} e^{\mathrm{Y}_{j}^{(r)}-\mathrm{Y}_{k}^{(r)}} \sum_{l \in \mathcal{I}}^{l \neq k} \frac{D_{r, l}}{D_{r, k}} e_{l}^{\mathrm{Y}_{l}^{(r)}-\mathrm{Y}_{k}^{(r)}}\right] \\
& =\frac{1}{D_{r, k}^{2}} \sum_{j \in \mathcal{I}} \sum_{l \in \mathcal{I}}^{l \neq j} D_{r, j} D_{r, l} E\left[e^{\mathrm{Y}_{j}^{(r)}-\mathrm{Y}_{k}^{(r)}+\mathrm{Y}_{l}^{(r)}-\mathrm{Y}_{k}^{(r)}}\right] \\
& =\frac{e^{4 \psi}}{D_{r, k}^{2}} \sum_{j \in \mathcal{I}} D_{r, j}^{2}+\frac{e^{3 \psi}}{D_{r, k}^{2}} \sum_{j \in \mathcal{I}}^{k \neq j} \sum_{l \in \mathcal{I}}^{l \neq k, j} D_{r, j} D_{r, l},
\end{aligned}
$$

where $\psi=2(1+\rho) \sigma^{2}$, with $\rho=1$ for the mono-static system setting and $\rho=0$ for the multi-static scenario. We then impose

$$
\left\{\begin{array} { l } 
{ \eta _ { \mathbf { X } _ { k } ^ { ( r ) } } = e ^ { \eta \mathrm { T } _ { k } ^ { ( r ) } + \frac { 1 } { 2 } \sigma _ { \mathrm { T } _ { k } ^ { ( r ) } } ^ { 2 } } } \\
{ \delta _ { \mathbf { X } _ { k } ^ { ( r ) } } = e ^ { 2 \eta _ { \mathrm { T } _ { k } ^ { ( r ) } } + 2 \sigma _ { \mathrm { T } _ { k } ^ { ( r ) } } ^ { 2 } } }
\end{array} \Rightarrow \left\{\begin{array}{l}
\eta_{\mathrm{T}_{k}^{(r)}}=\ln \frac{\eta_{\mathrm{x}_{k}^{(r)}}^{2}}{\sqrt{\delta \mathrm{X}_{k}^{(r)}}} \\
\sigma_{\mathrm{T}_{k}^{(r)}}^{2}=\ln \frac{\delta_{\mathbf{x}_{k}^{(r)}}}{\eta_{\mathrm{X}_{k}^{(r)}}^{2}}
\end{array}\right.\right.
$$


Then, the conditional probability in Eq. (11) can be approximated as

$$
p_{c, k}^{(r)} \approx \frac{\operatorname{Pr}\left(\mathrm{T}_{k}^{(r)}<\hat{\gamma}, \mathrm{S}_{f, i}>\hat{\gamma}_{h, i}, \forall i \in \mathcal{I}\right)}{\prod_{i \in \mathcal{I}}\left[1-F_{\mathrm{S}}\left(\hat{\gamma}_{h, i}\right)\right]},
$$

where we defined $\hat{\gamma}=-\ln \gamma$. To compute the approximated capture probability in Eq. (19) we first need to determine the covariance between $\mathrm{T}_{k}^{(r)}$ and $\mathrm{S}_{f, i}$, which we define as $c_{\mathbf{T}_{k}^{(r)}, i}=\operatorname{Cov}\left(\mathbf{T}_{k}^{(r)}, \mathrm{S}_{f, i}\right)$, for all $i \in \mathcal{I}$. To this aim we first compute the correlations

$$
\begin{aligned}
\delta_{\mathbf{X}_{k}^{(r), i}} & \triangleq E\left[\mathbf{X}_{k}^{(r)} e^{\mathrm{S}_{f, i}}\right]=E\left[\sum_{j \in \mathcal{I}}^{j \neq k} \frac{D_{r, j}}{D_{r, k}} e^{\Upsilon_{j}^{(r)}-Y_{k}^{(r)}} e^{\mathrm{S}_{f, i}}\right] \\
& =\frac{1}{D_{r, k}} \sum_{j \in \mathcal{I}}^{j \neq k} D_{r, j} E\left[e^{\mathbf{Y}_{j}^{(r)}-\mathbf{Y}_{k}^{(r)}+\mathrm{S}_{f, i}}\right] \\
& = \begin{cases}\frac{e^{\frac{1}{2}\left(\psi+\sigma^{2}\right)}}{D_{r, k}} \sum_{j \in \mathcal{I}}^{j \neq k} D_{r, j} & k=i \\
\frac{e^{\psi+\frac{1}{2} \sigma^{2}}}{D_{r, k}} \sum_{j \in \mathcal{I}}^{j \neq k, j \neq i} D_{r, j}+\frac{D_{r, i}}{D_{r, k}} e^{\frac{1}{2}\left(3 \psi+\sigma^{2}\right)} & k \neq i .\end{cases}
\end{aligned}
$$

Being $E\left[e^{\mathrm{T}_{k}^{(r)}} e^{\mathrm{S}_{f, i}}\right]=E\left[e^{\mathrm{T}_{k}^{(r)}}\right] E\left[e^{\mathrm{S}_{f, i}}\right] e^{c_{\mathrm{T}_{k}(r), i}}$, with $i \in \mathcal{I}$, the covariance between $\mathrm{T}_{k}^{(r)}$ and the fading component on the $i$-th forward link is thus obtained as

$$
c_{\mathbf{T}_{k}^{(r)}, i}=\ln \frac{\delta_{\mathbf{X}_{k}^{(r)}, i}}{e^{\eta_{\mathrm{T}_{k}^{(r)}}+\frac{1}{2}\left(\sigma_{\mathrm{T}_{k}^{(r)}}^{2}+\sigma^{2}\right)}} .
$$

The computation of probabilities in Eqs. (14) and (19) requires multivariate integration that can be cumbersome when the cardinality of $\mathcal{I}$ is high. Nevertheless, due to the presence of channel access coordination at MAC layer, the number of contending tags during colliding slots is reasonably small. Moreover, the approximated idle and capture probabilities are expressed in terms of multivariate Gaussian CDF and can be efficiently evaluated by means of standard numeric integration approaches [42].

We remark that the proposed approximation approach is an extension of the generalized Fenton-Wilkinson (F-W) method proposed in [38]. It has been shown that F-W method provides a low accuracy for $\sigma>4 \mathrm{~dB}$ [43]. Nevertheless, RFID applications are typically intended for indoor scenarios where $\sigma$ is generally small. Moreover, in the specific case considered in this paper, we are interested in approximating the tail distribution of a sum of log-normal random variables and F-W approach provides a good accuracy even for larger values of $\sigma$ [44]. Finally, we highlight that the described method is well suited to approximate randomly weighted sums of log-normal random variables [38]. Then, the proposed rationale can be used to provide accurate approximation even in scenarios characterized by more general product channels, which includes different fading statistics (e.g. Exponential distribution for Rayleigh fading, Non-central Chi-square distribution for Rice fading, Gamma distribution for Nakagami fading, etc.). More precisely, regardless of the fading statistics considered for the single two-way channel, the interference and the SIR are approximated by a log-normal random variable with proper parameters. The inclusion of additional random components in Eq. (2) would clearly affect the first and second order moment of $\mathrm{Z}^{(r)}$ and $\mathrm{X}_{k}^{(r)}$ and, in turn, the parameters of the approximating log-normal random variable. Nevertheless, this effect can be easily accounted in our framework by varying $\sigma$.

\section{Enhanced Gen 2 Protocol}

\section{A. Effects of Capture Diversity}

In the multi-static system scenario considered in this paper the signal detection occurs at different spatial locations and the backscattering links from tags to detection points are characterized by both different path losses and different fading components. Consequently, during a multiple tags' reply the power of interfering signals is different at different receivers. Let us consider a given tag $k$ and two given detection points $r$ and $p$, the sample of the SIR is different at the considered receivers, that is $\operatorname{SIR}_{k}^{(r)} \neq \mathrm{SIR}_{k}^{(p)}$, with $r \neq p$. This implies that each tag has a different chance to be captured within the readers' network. Equivalently, the capture probability distributes differently over the set of interfering tags $\mathcal{I}$ at different detection points.

An illustration of potential benefits of capture diversity is shown in Fig. 2 for a multi-static system with $R=3$ receivers. The capture probabilities for each tag at each receiver are computed by using Eq. (11) for a randomly generated spatial scenario; moreover, two values $\sigma=2 \mathrm{~dB}$ (Fig. 2-(a)) and $\sigma=6$ dB (Fig. 2-(b)) are considered and a slot with 5 replying tags is assumed. Fig. 2-(a) clearly shows that different tags have a different chance of being correctly identified at different detection points despite the interference. Observe that the standard deviation $\sigma$ impacts on how the capture probabilities distribute among receivers. As expected, a larger $\sigma$ induces more dispersion but, interestingly, the peaks in the capture distribution are not substantially affected. This can be explained by observing that the capture probability is also affected by the relative distance between the interfering tags and the detection points.

This relevant feature of multi-static RFID systems that we denote as capture diversity can be conveniently exploited to boost-up the identification process performance. Indeed, thanks to the capture diversity, different tags can be successfully identified by different receivers during those slots experiencing multiple tags' replies. Note that, similarly to conventional antenna diversity approaches, spatial correlation may negatively impact the performance of the diversity mechanism, reducing the chance of successfully identifying tags simultaneously. Including a correlation structure to the channel abstraction would not require substantial modifications to our framework. However, we believe that assuming i.i.d. fading components still provides a representative insights on the maximum achievable diversity gain in the considered settings and the qualitative impact of channel conditions and deployment.

\section{B. Proposed Protocol Modifications}

To take significant advantages of capture diversity, only slight modifications are needed to the centralized channel 


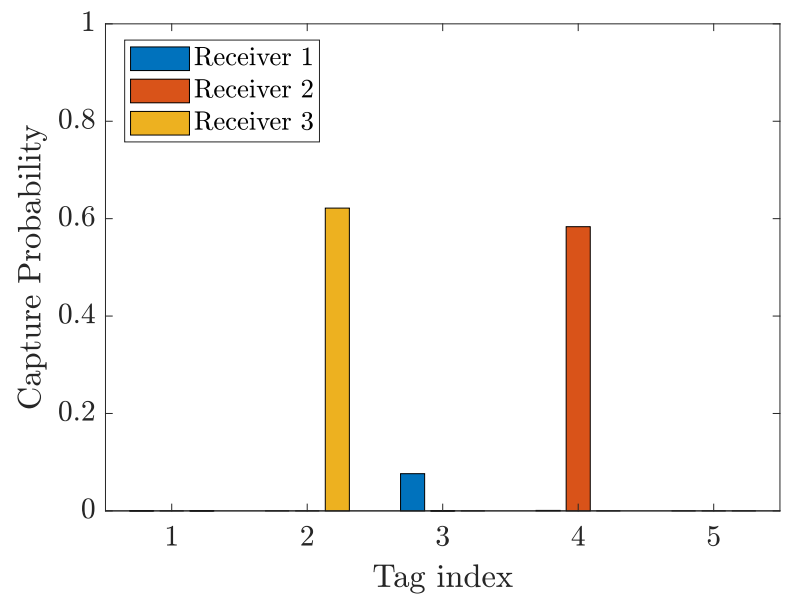

(a)

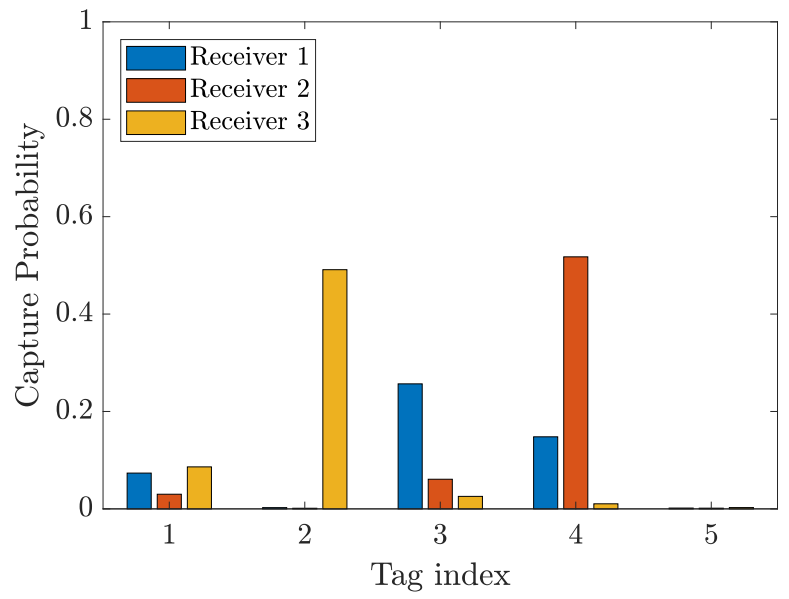

(b)

Fig. 2. Capture probabilities for a slot with $|\mathcal{I}|=5$ replying tags and $R=3$. (a) $\sigma=2 \mathrm{~dB}$. (b) $\sigma=6 \mathrm{~dB}$.
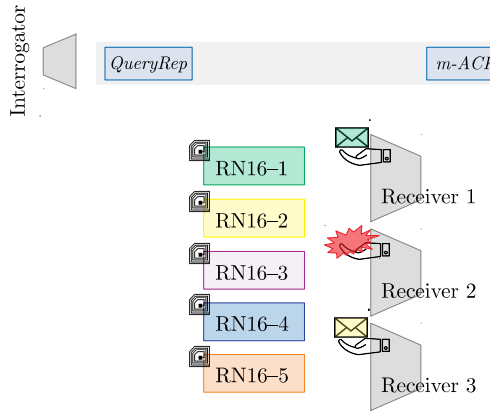

(a)

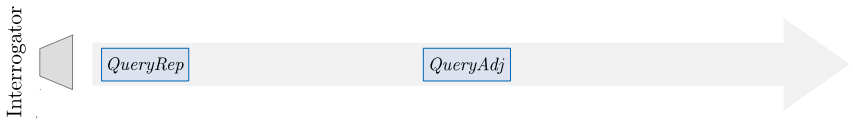

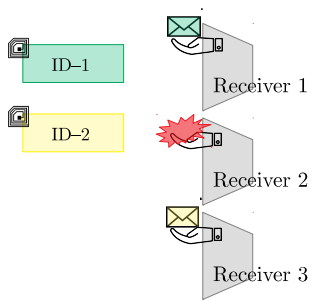

ren

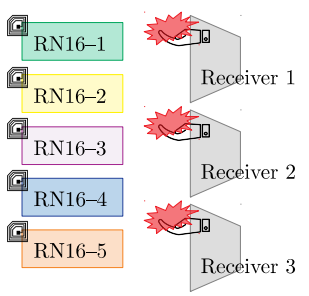

(b)

Fig. 3. Illustration of the modified MAC protocol during a generic slot with multiple replying tags. (a) Example of multiple tags identification allowed by capture diversity. (b) Example of a slot resulting in a collision.

access scheme described in Section II-B, under the assumption that the latency introduced by the readers' network is negligible. In particular, assuming that the channel attenuation is constant within a frame duration (i.e., $2^{Q}$ slots), the capture probability is time-invariant within the frame. Then, the interrogator can force all the interfering tags to communicate their IDs so as their responses can be captured at the different detection points. This can be achieved by a modified ACK command $(m-A C K)$ that instructs all the tags in $\mathcal{I}$ to communicate the relative IDs.

Fig. 3-(a) illustrates the concept of capture diversity through an example of a multiple reply resolved as a successful identification of multiple tags in the distributed system with three readers considered above. Specifically, a multiple reply with five interfering tags is considered (i.e., five tags have a null $\mathrm{SN}$ in the considered slot). The RN16 of the first tag (RN16-1) is captured by Receiver 1, the RN16 of the second tag (RN16-2) is captured by Receiver 3, whereas the remaining tags' signals fail to be captured. Note also that Receiver 2 perceives a collision. In response to this event, the interrogator issues a $m-A C K$ command forcing all the tags to transmit their IDs. Given that the capture probability is constant over a fading block, Receiver 1 and Receiver 3 are able to correctly decode the identifiers associated with the previously captured tags. We remark that the other tags also communicate their ID, however they can not be captured in the receivers' network. It is worth mentioning that the same tag's signal can be captured by different detection points. Nevertheless, in that event, the redundant identifications may be simply ignored, although this information may be useful for other purposes, e.g. tag localization, which is not considered herein.

Finally, Fig. 3-(b) shows an example of a multiple reply resulting in a collision, where no tag is successfully identified. The collision occurs with probability

$$
p_{c}=\prod_{r=1}^{R} p_{c}^{(r)},
$$

where $p_{c}^{(r)}$ is the collision probability measured at the $r$ th receiver. In that case, the channel contention mechanism simply evolves as for a centralized single-reader system. Observe that, although a non-zero probability of experimenting a collision event exists, collision occurrence is substantially reduced in distributed systems given the presence of redundant detection points that "see" a different propagation channel.

The implementation of the proposed acknowledgement 
TABLE I

MAIN SIMULATION PARAMETERS

\begin{tabular}{|c|c|}
\hline Parameter & Value \\
\hline$P_{\mathrm{tx}}$ & $2 \mathrm{~W}$ \\
\hline$f$ & $915 \mathrm{MHz}$ \\
\hline$\gamma_{d}$ & $10 \mathrm{pW}$ \\
\hline$\gamma_{h}$ & $10 \mu \mathrm{W}$ \\
\hline$\gamma$ & $6 \mathrm{~dB}$ \\
\hline
\end{tabular}

strategy does require neither receivers distributed control nor receivers cooperation algorithms. Indeed, each receiver performs tags identification independently without the need of shared information from other receivers. Only limited information sharing between the receivers and the interrogator is required in order to report IDs of identified tags. This occurs through a separate control channel mapped over the receivers' network. Consequently, the described protocol modification does not imply substantial communication overhead and can be supported with limited system complexity. It is also worth noting that the described diversity scheme differs from conventional diversity concept, where signal redundancy is exploited by means of combining approaches that aims at maximizing detection performance. Instead, our approach exploits the inherent spatial diversity offered by the multi-receiver architecture in combination with multiple access negotiation strategy. As so, our solution does not require signal combining (e.g. Maximal Ratio Combining (MRC)) policies and, in turn channel estimation. The exploitation of a classic combining technique would require higher communication overhead from the receivers to the interrogator and, in general, would not allow the identification of multiple tags simultaneously.

\section{Numerical Results}

In this section, we present a set of results that allow to quantify the benefits achievable by exploiting capture diversity under our enhanced Gen 2 protocol in multi-static RFID system scenarios.

We considered a multi-static scenario with $N=10$ tags uniformly disseminated at fixed spatial locations within a circular region around the interrogator. The tag population cardinality is expressly kept small since the Q-algorithm is designed so that after an initial training period, the size of the contention window quickly adapts to the tag population. Therefore, the number of multiple replies in single slots is expected to stabilize to a small number regardless of the tag population size. The detection points are placed at the same distance $d_{I R}$ from the interrogator. We remark that, although a single spatial configuration is considered, the chosen scenario is representative. Indeed, despite the fixed spatial dissemination of tags, the selection of replying tags during the identification process is random due to the presence of MAC anti-collision scheme. Simulation parameters are listed in Tab. I. We refer to the $f m 0$ physical layer defined by the standard and the relative timing parameters are listed in Tab. II. Further detail on $f m 0$ physical layer can be found in [5] and are omitted here due to space limitations.

In order to quantify the impact of capture diversity on the MAC collision resolution capabilities, we define the Capture
TABLE II

$f m 0$ TIMING PARAMETERS

\begin{tabular}{llc}
\hline Command & Event & Slot duration $[\mu \mathrm{s}]$ \\
\hline \hline \multirow{3}{*}{ QueryRep } & Idle & 180 \\
& Success & 1500 \\
& Collision & 310 \\
\hline \multirow{3}{*}{ QueryAdj } & Idle & 260 \\
& Success & 1600 \\
& Collision & 390 \\
\hline
\end{tabular}

Rate (CR) as the fraction of multiple tag replies resolved as successful identifications due to capture effect. Observing that each powering event, $\mathcal{P}_{\mathcal{A}}$, corresponds to an interrogation session where only $|\mathcal{A}|$ tags contend the channel, the expected capture rate, which we denote as $\bar{R}_{C}$, can be derived by marginalization with respect to the powering events as

$$
\bar{R}_{C}=\sum_{\lambda \subset \Lambda} E\left[C R \mid \mathcal{P}_{\mathcal{A}}\right] \operatorname{Pr}\left(\mathcal{P}_{\mathcal{A}}\right) .
$$

Furthermore, to measure the identification performance we refer to the Tags Identification Speed (TIS) [45], defined as the number of successfully identified tags per second. The expected TIS, denoted by $\bar{R}_{T}$, can be determined as

$$
\bar{R}_{T}=\sum_{\mathcal{A} \subset \mathcal{T}} E\left[T I S \mid \mathcal{P}_{\mathcal{A}}\right] \operatorname{Pr}\left(\mathcal{P}_{\mathcal{A}}\right) .
$$

To obtain the quantities in Eqs. (24) and (23) we enforce a semi-analytic approach where the conditional expectations $E\left[C R \mid \mathcal{P}_{\mathcal{A}}\right]$ and $E\left[T I S \mid \mathcal{P}_{\mathcal{A}}\right]$ are obtained via Monte-Carlo simulation, whereas the probabilities related to the channel statistics are derived by MMA as described in the previous sections. Specifically, the TIS, conditioned on each powering event, is averaged over 1e3 Monte-Carlo runs and the marginalization is obtained using $\operatorname{Pr}\left(\mathcal{P}_{\mathcal{A}}\right)$ in Eq. (5).

To benchmark our analysis, we compare the performance of the proposed generic multi-static system with a classic monostatic system, where a single reader acts as both interrogator and detection point, and a bi-static system, where a single detection point is physically separated from the interrogator. Fig. 4 shows the capture rate as a function of the number of deployed receivers $R$ for different values of $\sigma$, with $c=0.5$. As expected, $\bar{R}_{C}$ increases with $R$ as a consequence of the increase of diversity order in the system. The bi-static system (i.e., multi-static with $R=1$ ) provides a slight enhancement of the performance in terms of capture rate compared to the mono-static system, as detecting the tag at a dislocated position increases the chance that other active tags are captured. Interestingly, in a mono-static system, the capture rate is larger with more severe fading conditions (i.e., $\sigma=6$ ). This is explained considering that in a mono-static system, fading affects the powering event and the detection event for a given tag in the same way, since forward and backscattering links are fully correlated. A higher $\sigma$ corresponds to a higher variability of the fading realizations among different tags, which is beneficial for the capture probability. When the detection point is not co-located, forward and backscattering links are independent and an increase of $\sigma$ determines also a reduction of the probability of correct detection of each of the 


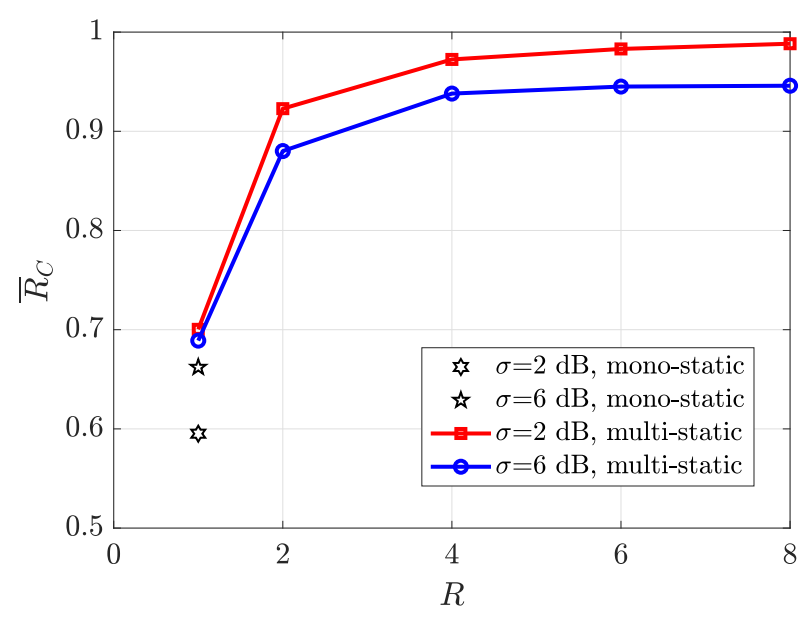

Fig. 4. Capture rate, $\bar{R}_{C}$, as a function of the number of receivers $R$ for different values of $\sigma$

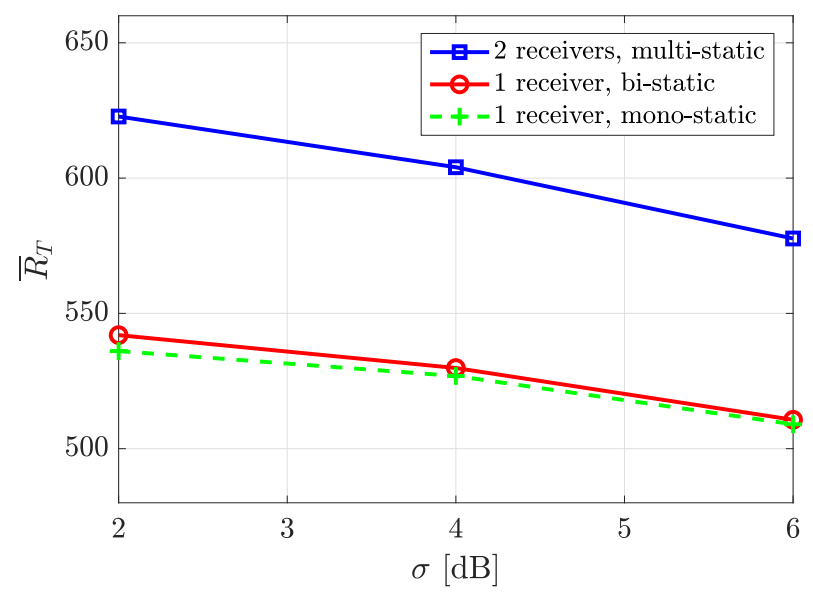

Fig. 5. Tag identification speed, $\bar{R}_{T}$, as a function of $\sigma$.

powered tag. Finally, a substantial improvement of the capture rate is achieved with multiple receivers when compared to the mono-static and the bi-static system.

In Fig. 5, we report the TIS as a function of $\sigma$, comparing the case of single interrogator colocated with the detection point (mono-static system), the bi-static system, and the multistatic case with two receivers that are physically separated from the interrogator. The granularity parameter of the anticollision protocol is $c=0.5$. It can be seen that, in the considered scenario, the physical separation between the interrogator and the detection point only yields slight performance improvement with respect to the mono-static architecture. Differently, the deployment of an additional detection point guarantees a substantial improvement in the TIS that is justified by the exploitation of capture diversity and the resulting increase of the capture rate. When dealing with the TIS, it is clear that an increase of $\sigma$ introduces a penalty in the performance, mainly because it reduces the tags' powering probability.

In Fig. 6, we report the TIS as a function of the number of deployed detection points $R$, for different values of $\sigma$ and the granularity parameter of the anti-collision protocol

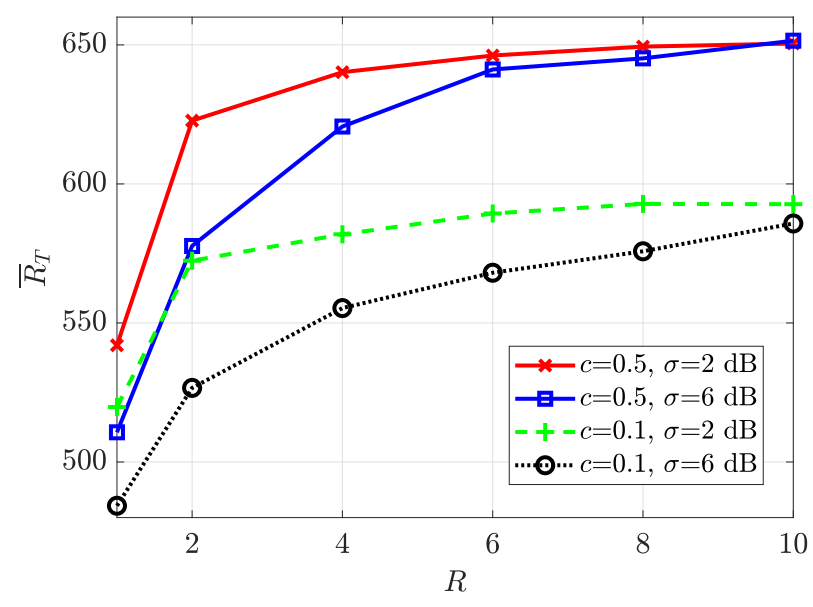

Fig. 6. Tag identification speed, $\bar{R}_{T}$, as a function of number of receivers $R$.

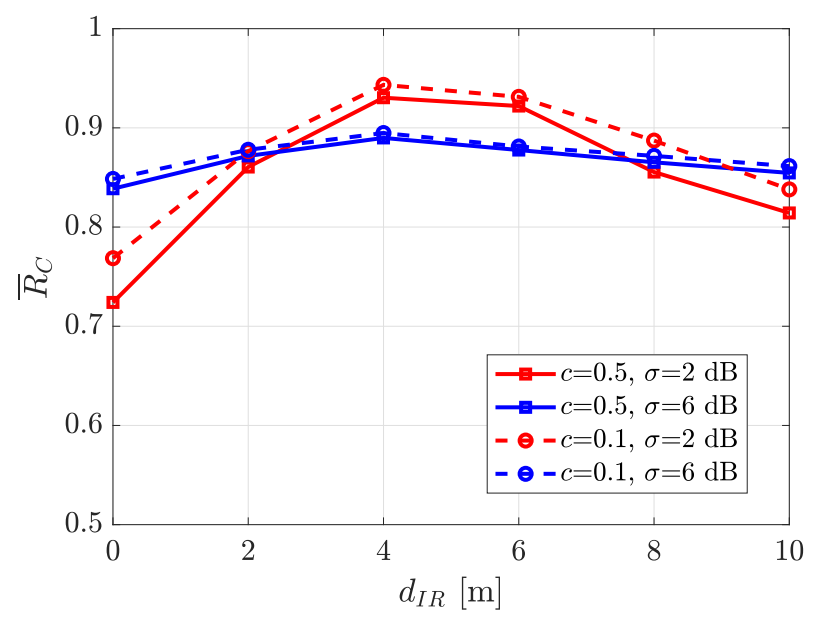

Fig. 7. Capture rate, $\bar{R}_{C}$, as a function of interrogator-to-reader distance for $R=2$.

c. As expected, the identification performance increases as $R$ increases. Nevertheless, as already observed for the capture rate, the performance gain tends to saturate as the number of considered detection points increases, suggesting that substantial TIS improvement can be achieved by deploying only a few additional receivers, thus significantly containing the system complexity. It is also evident that a higher TIS is obtained with $c=0.5$ for all the considered values of the $\sigma$. This suggests that it is beneficial to choose an aggressive frame update policy to better exploit the performance boost up offered by the capture diversity.

The impact of the distance $d_{I R}$ between the interrogator and the detection points is analyzed in Fig. 7 for different values of $c$ and $\sigma$, while assuming $R=2$. It can be seen that, in the considered deployment, $\bar{R}_{C}$ exhibits a maximum around $d_{I R}=4$ for both the considered values of $\sigma$. However, the capture rate drops as the $d_{I R}$ increases too much. Note also that the capture rate is less sensible to the interrogator-toreceiver distance when $\sigma=6 \mathrm{~dB}$ and, as expected, is almost independent of the granularity parameter $c$. Fig. 8 reports the TIS as a function of $d_{I R}$ for different values of $\sigma$ and 


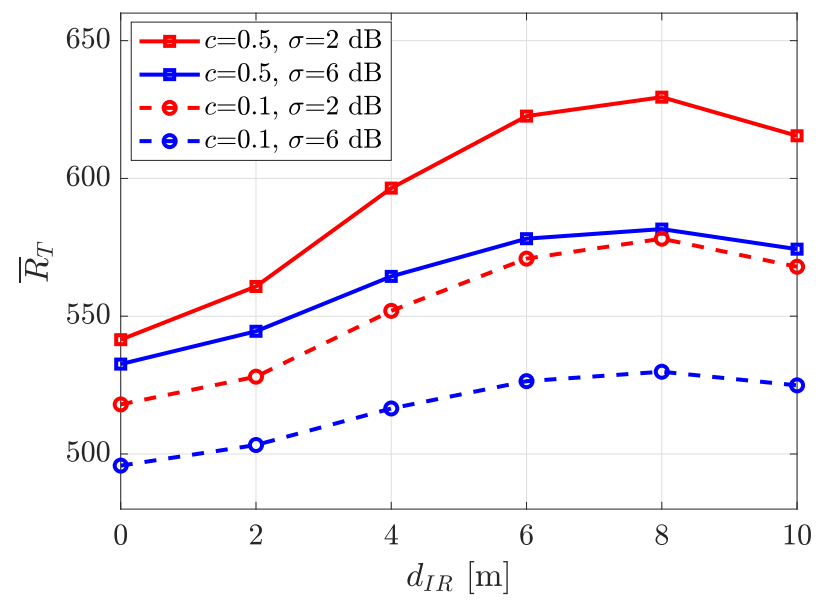

Fig. 8. Tag identification speed, $\bar{R}_{T}$, as a function of the distance between interrogator and receivers for $R=2$.

$c$, when $R=2$. As expected, the trend of the capture rate reflects on the TIS, which exhibits a maximum and then drops as $d_{I R}$ increases above 4 meters. Again, better identification performance are obtained by setting $c=0.5$. In the considered scenario, the observed dependence on the distance can be easily justified by observing that, when the distance $d_{I R}$ increases, the differences in relative distances between tags and detection points increase as well, thus emphasizing capture diversity. This effect clearly dismisses as $d_{I R}$ increases too much, given that average distances tends to increase and the probability of miss-detection becomes prominent. Observe that, Although there is a correlation between an increase of the capture rate and the probability of successful identification event, this is not the only trend that affects the TIS. Indeed, the TIS incorporates also miss-detection events during single tag replies, idle slots, and relative duration. Therefore, a particular parameter setting that maximizes the capture rate does not necessarily maximizes the TIS. The capture event probability does not distinguish whether the capture happens at one reader for a single tag or simultaneously at multiple readers for different tags. Since simultaneous multiple tag identification more likely occurs when readers are set apart from each other, the optimal distance between readers results to be larger when considering the TIS metric compared to the capture rate.

Finally, to validate the proposed modification to the acknowledgment policy, we compare our solution to the standard Gen 2 algorithm. In Fig. 9, we show the results of the comparison in terms of TIS, for $c=0.5$. It is shown how the exploitation of capture diversity achieved by the proposed solution yields a substantial performance improvement if compared to the legacy Gen 2 acknowledgment signaling. This is due to the fact that the standard Gen 2 acknowledgment structure only allows for the identification of a single tag in each successful slot. Indeed, the acknowledgment packet contains the RN16 of the captured tag, which forces only that tag to transmit the EPC identification code. On the contrary, our proposal allows for multiple concurrent identifications at different detection points, yielding a consistent boost of the TIS.

It can also be seen that the performance gap is exacerbated

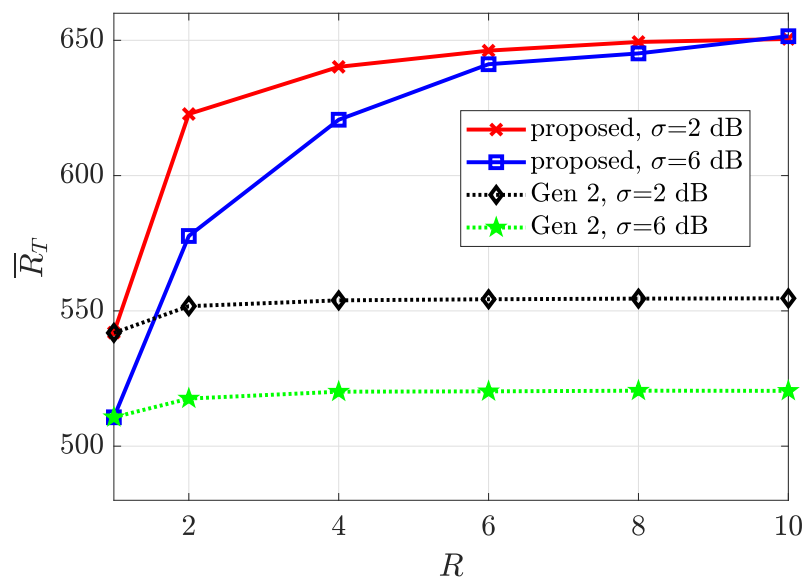

Fig. 9. Tag Identification speed, $\bar{R}_{T}$, as a function of $R$ for $c=0.5$. Comparison between the proposed solution and the Gen 2 standard acknowledgment policy.

as the number of deployed detection points increases. More precisely, under the standard approach, the TIS exhibits very low sensitivity to $R$ and the saturation is already obtained for $R=4$. Again, this behavior is related to the inadequacy of Gen 2 to fully exploit the propagation channel features.

\section{CONCLUSions}

In this paper we presented a complete framework for the characterization of multiple access interference and capture effect on the performance metrics for tag identification in multi-static RFID scenarios. We showed a substantial performance improvement in the presence of multiple distributed detection points that can exploit receiver diversity in terms of capture probability. Furthermore, we proposed a modification to the standard Gen 2 anti-collision protocol to support the multi-static scenario and enable multiple receptions at different detection points. The performance gain is relevant even with a small number of receivers, making our solution competitive in terms of complexity and cost in the RFID system landscape. We believe that the proposed analysis lays the foundations for accurate modeling and design of proper protocol and architectural solutions in the field of RF identification applications and the overall massive IoT arena as well.

\section{REFERENCES}

[1] C. Xu, L. Yang, and P. Zhang, "Practical backscatter communication systems for battery-free internet of things: A tutorial and survey of recent research," IEEE Signal Processing Magazine, vol. 35, no. 5, pp. 16-27, 2018.

[2] R. Duan, X. Wang, H. Yigitler, M. U. Sheikh, R. Jantti, and Z. Han, "Ambient backscatter communications for future ultra-low-power machine type communications: Challenges, solutions, opportunities, and future research trends," IEEE Communications Magazine, vol. 58, no. 2, pp. 42-47, February 2020.

[3] F. Zheng and T. Kaiser, Digital Signal Processing for RFID. John Wiley \& Sons, 2016.

[4] P. V. Nikitin and K. V. S. Rao, "Performance limitations of passive UHF RFID systems," in 2006 IEEE Antennas and Propagation Society International Symposium, July 2006, pp. 1011 - 1014.

[5] Standard, "EPC Radio-Frequency Identity Protocols Generation-2 UHF RFID," 2013.

[6] L. Sanchez and V. Ramos, "Towards an Efficient Identification Process for Large-Scale RFID Systems," Sensors, vol. 18, no. 7, p. 2350, 2018. 
[7] A. Fahim, T. Elbatt, A. Mohamed, and A. Al-Ali, "Towards Extended Bit Tracking for Scalable and Robust RFID Tag Identification Systems," IEEE Access, vol. 6, pp. 27 190-27 204, 2018.

[8] M. Lee, J. Wang, H. Li, F. Ye, and H. Yue, "Reader Scheduling for Information Collection in Large-Scale RFID Systems," in 2018 IEEE 88th Vehicular Technology Conference (VTC-Fall), Aug 2018, pp. 1-5.

[9] M. Ma, P. Wang, and C. Chu, "Redundant Reader Elimination in LargeScale Distributed RFID Networks," IEEE Internet of Things Journal, vol. 5, no. 2, pp. 884-894, April 2018.

[10] N. Van Huynh, D. T. Hoang, X. Lu, D. Niyato, P. Wang, and D. I. Kim, "Ambient backscatter communications: A contemporary survey," IEEE Communications Surveys \& Tutorials, vol. 20, no. 4, pp. 2889-2922, 2018.

[11] D. Darsena, "Noncoherent detection for ambient backscatter communications over OFDM signals," IEEE Access, vol. 7, pp. 159415-159425, 2019.

[12] C. Boyer and S. Roy, "Space time coding for backscatter RFID," IEEE Transactions on Wireless Communications, vol. 12, no. 5, pp. 2272 2280, May 2013.

[13] C. He, Z. J. Wang, and V. C. M. Leung, "Unitary query for the $m \times$ $l \times n$ MIMO backscatter RFID channel," IEEE Transactions on Wireless Communications, vol. 14, no. 5, pp. 2613-2625, 2015.

[14] C. He, Z. J. Wang, C. Miao, and V. C. M. Leung, "Block-level unitary query: Enabling orthogonal-like space-time code with query diversity for MIMO backscatter RFID,' IEEE Transactions on Wireless Communications, vol. 15, no. 3, pp. 1937-1949, 2016.

[15] C. He, Z. J. Wang, and C. Miao, "Query diversity schemes for backscatter RFID communications with single-antenna tags," IEEE Transactions on Vehicular Technology, vol. 66, no. 8, pp. 6932-6941, 2017.

[16] D. Mishra and E. G. Larsson, "Monostatic backscattering detection by multiantenna reader," in 2019 53rd Asilomar Conference on Signals, Systems, and Computers, 2019, pp. 697-701.

[17] —, "Multi-tag backscattering to MIMO reader: Channel estimation and throughput fairness," IEEE Transactions on Wireless Communications, vol. 18, no. 12, pp. 5584-5599, 2019.

[18] F. Hessar and S. Roy, "Energy based performance evaluation of passive EPC Gen 2 Class 1 RFID systems," IEEE Transactions on Communications, vol. 61, no. 4, pp. 1337-1348, 2013.

[19] H. Salah, H. A. Ahmed, J. Robert, and A. Heuberger, "A time and capture probability aware closed form frame slotted ALOHA frame length optimization," IEEE Communications Letters, vol. 19, no. 11, pp. 2009-2012, Nov 2015.

[20] J. J. Alcaraz, J. Vales-Alonso, E. Egea-López, and J. Garcia-Haro, "A stochastic shortest path model to minimize the reading time in DFSAbased RFID systems," IEEE Communications Letters, vol. 17, no. 2, pp. 341-344, 2013.

[21] B. Li and J. Wang, "Efficient Anti-Collision Algorithm Utilizing the Capture Effect for ISO 18000-6C RFID Protocol,' IEEE Communications Letters, vol. 15, no. 3, pp. 352-354, March 2011.

[22] H. Wu and Y. Zeng, "Passive RFID Tag Anticollision Algorithm for Capture Effect," IEEE Sensors Journal, vol. 15, no. 1, pp. 218-226, Jan 2015.

[23] A. U. H. Sheikh, Y. . Yao, and X. Wu, "The ALOHA systems in shadowed mobile radio channels with slow or fast fading," IEEE Transactions on Vehicular Technology, vol. 39, no. 4, pp. 289-298, 1990

[24] R. Valentini, R. Alesii, M. Levorato, and F. Santucci, "Cross-layer analysis of RFID systems with correlated shadowing and random radiation efficiency," in 2019 IEEE International Conference on Communications, ICC 2019, 2019, pp. 1-7.

[25] P. D. Marco, F. Santucci, and C. Fischione, "Modeling anti-collision protocols for RFID Systems with multiple access interference," in 2014 IEEE International Conference on Communications (ICC), June 2014 pp. 5938-5944.

[26] Y. H. Al-Badarneh, M. S. Alouini, and C. N. Georghiades, "Performance analysis of monostatic multi-tag backscatter systems with general order tag selection," IEEE Wireless Communications Letters, vol. 9, no. 8, pp. 1201-1205, 2020.

[27] J. Waldrop, D. W. Engels, and S. E. Sarma, "Colorwave: a MAC for RFID reader networks," in 2003 IEEE Wireless Communications and Networking, 2003. WCNC 2003., vol. 3, 2003, pp. 1701-1704 vol.3.

[28] S. Tang, J. Yuan, X. Li, G. Chen, Y. Liu, and J. Zhao, "Raspberry: A stable reader activation scheduling protocol in multi-reader RFID systems," in 2009 17th IEEE International Conference on Network Protocols, 2009, pp. 304-313.

[29] S. Tang, C. Wang, X. Li, and C. Jiang, "Reader activation scheduling in multi-reader RFID systems: A study of general case," in 2011 IEEE
International Parallel Distributed Processing Symposium, 2011, pp. 1147-1155.

[30] Z. Zhou, H. Gupta, S. R. Das, and X. Zhu, "Slotted scheduled tag access in multi-reader RFID systems," in 2007 IEEE International Conference on Network Protocols, 2007, pp. 61-70.

[31] A. Mohsenian-Rad, V. Shah-Mansouri, V. W. S. Wong, and R. Schober, "Distributed channel selection and randomized interrogation algorithms for large-scale and dense RFID systems," IEEE Transactions on Wireless Communications, vol. 9, no. 4, pp. 1402-1413, 2010

[32] L. Yang, Y. Qi, J. Han, C. Wang, and Y. Liu, "Shelving interference and joint identification in large-scale RFID systems," IEEE Transactions on Parallel and Distributed Systems, vol. 26, no. 11, pp. 3149-3159, 2015.

[33] J. Ho, D. W. Engels, and S. E. Sarma, "HiQ: a hierarchical q-learning algorithm to solve the reader collision problem," in International Symposium on Applications and the Internet Workshops (SAINTW'06), 2006, pp. 4 pp. -91

[34] X. Liu, J. Yin, S. Zhang, B. Xiao, and B. Ou, "Time-efficient target tags information collection in large-scale RFID systems," IEEE Transactions on Mobile Computing, pp. 1-1, 2020.

[35] P. N. Alevizos, K. Tountas, and A. Bletsas, "Multistatic scatter radio sensor networks for extended coverage," IEEE Transactions on Wireless Communications, vol. 17, no. 7, pp. 4522-4535, 2018.

[36] M. Ouroutzoglou, G. Vougioukas, P. N. Alevizos, A. G. Dimitriou, and A. Bletsas, "Multistatic Gen2 RFID over ethernet with commodity sdrs," in 2019 IEEE International Conference on RFID Technology and Applications (RFID-TA), 2019, pp. 393-398.

[37] R. Valentini, P. di Marco, R. Alesii, and F. Santucci, "Exploiting capture diversity in distributed passive RFID systems," in 2020 10th Annual Computing and Communication Workshop and Conference (CCWC), 2020, pp. 0996-1000.

[38] M. Pratesi, F. Santucci, and F. Graziosi, "Generalized moment matching for the linear combination of lognormal rvs: application to outage analysis in wireless systems," IEEE Transactions on Wireless Communications, vol. 5, no. 5, pp. 1122-1132, 2006

[39] R. Valentini, P. D. Marco, R. Alesii, and F. Santucci, "Cross-layer analysis of distributed passive RFID systems over faded backscattering links," in 2020 IEEE Wireless Communications and Networking Conference (WCNC), 2020, pp. 1-6.

[40] N. C. Karmakar, Handbook of smart antennas for RFID systems. John Wiley \& Sons, 2011

[41] M. C. Jeruchim, P. Balaban, and K. S. Shanmugan, Simulation of communication systems: modeling, methodology and techniques. Springer Science \& Business Media, 2006.

[42] A. Genz, "Numerical computation of multivariate normal probabilities," Journal of Computational and Graphical Statistics, vol. 1, no. 2, pp. 141-149, 1992

[43] S. C. Schwartz and Y. S. Yeh, "On the distribution function and moments of power sums with log-normal components," The Bell System Technical Journal, vol. 61, no. 7, pp. 1441-1462, 1982.

[44] G. L. Stüber, Principles of mobile communication. Springer, 2001, vol. 2.

[45] C. Wang, M. Daneshmand, K. Sohraby, and B. Li, "Performance analysis of RFID generation-2 protocol," IEEE Transactions on Wireless Communications, vol. 8, no. 5, pp. 2592-2601, 2009 